\title{
The Electromagnetic Energy-Momentum Tensor in Expanding Universes
}

\author{
Hans-J. Fahr ${ }^{1 *}$ and Michael Heyl ${ }^{2}$ \\ ${ }^{1}$ Argelander Institut für Astronomie, Universität Bonn \\ Auf dem Hügel 71, 53121 Bonn, Germany \\ ${ }^{2}$ Deutsches Zentrum für Luft und Raumfahrt (DLR) \\ Königswinterer Str. 522 - 524, 53227 Bonn, Germany \\ Email: hfahr@astro.uni-bonn.de
}

\begin{abstract}
In two previous papers the authors have investigated the characteristics of freely propagating cosmic photons in expanding homogenous flat universes from particle and wave points of view, respectively. The result was that the energy density of these freely propagating photons scales with $1 / S^{3}$ instead with the generally accepted and redshift-related $1 / S^{4}$, with $\mathrm{S}$ being the cosmic scale factor. This is a surprising result at first glance but finally very understandable if energy conservation has to be required also for cosmic photons without creating a conflict with observations. In this paper we treat freely propagating cosmic photons with a totally different approach, namely with the electromagnetic energy-momentum tensor (from now on abbreviated with EMT). The implementation of the EMT, when correctly interpreted for freely propagating photons, into Einstein's field equations then automatically leads to the same $1 / S^{3}$-scaling law for the energy density of cosmic photons, herewith confirming the results found earlier for the particle and wave view. We conclude that the observed cosmological redshift of photons (energy density $\left.\propto 1 / S^{4}\right)$ is compatible with the law of energy conservation, i.e energy density $\propto 1 / S^{3}$ if interpreted on a new physical basis.
\end{abstract}

Keywords: Cosmic microwave background, electromagnetic energy-momentum tensor, theory

\section{Introduction}

The phenomenon of the cosmological redshift has just recently been re-discussed by [1] and [2]. The result of both investigations was an energy density scaling of freely propagating cosmic photons according to $1 / S^{3}$, no matter how photons are described, i.e. as particles or as waves. The retrieved $1 / S^{3}$-scaling seems to be in conflict with the standard view of the cosmological redshift which predicts an energy density $\propto 1 / S^{4}$. This discrepancy, however, is not a serious but can be resolved as we show here.

In the particle view it is the change of the beat of the global world time over the travel time between the emission and absorption of the photons which makes the photons - when judged by a local clock appear with a reduced frequency (i.e. energy loss) and which finally leads to the observation of an energy density scaling according to $1 / S^{4}$ even though in the photon system the frequency is unaffected by the cosmic expansion and thus remains constant. The lower energy of the photon is just an observational effect. In the wave view the photon wavelength is stretched linearly with the expansion of the universe. The mode energy, however, remains constant and is just redistributed along an increasingly elongated wave mode. Thus, the observer again sees an energy density $\propto 1 / S^{4}$ while the total energy of the wave is conserved and leads to an energy density of $1 / S^{3}$.

Both perspectives are conciliatory with the law of energy conservation on one hand, but also with observational facts on the other hand. We now follow another way to investigate the cosmological redshift. This method will be based on the electromagnetic energy-momentum tensor EMT which must be applied to a homogenous isotropic flat universe.

\section{The GTR Electromagnetic Energy-Momentum Tensor}

The following study we start with the derivation of the energy-momentum tensor $T_{\nu \mu}$ for a pure photon universe, however, derived from its very basic roots, i.e. the electro-magnetic field tensor $F_{\nu \kappa}$ describing 
the cosmic electromagnetic radiation field. Usually (see e.g. [3]) one, instead of doing so, starts from the assumption that the cosmic radiation field, in view of its origin from the cosmic recombination era where baryons and photons still where in thermodynamical equilibrium, arises as and also continues staying a Planck radiation field for which energy density $\epsilon_{\nu}$ and radiation pressure $p_{\nu}$ can be connected to each other by the LTE- relation $\epsilon_{\nu}=T \cdot d p_{\nu} / d T-p_{\nu}$ valid in this case. Here we do want, however, to start from the very basic grounds of electromagnetic radiation fields whatever may be their nature and their spectrum (i.e. LTE or non-LTE ). Thus we start from the classical requirement that the electromagnetic forces $f_{\nu}$ can be derived as the $4 \mathrm{~d}$ - divergence of the underlying STR electromagnetic EMT, $T_{\nu \mu}$, in the form

$$
f_{\nu}=\sum_{\mu=1}^{\mu=4} \frac{\partial}{\partial x_{\mu}} T_{\nu \mu}
$$

From that relation one can conclude that the form of the covariant tensor $T_{\nu \mu}$ that we are looking for must be given by (e.g. see [4] or [5])

$$
T_{\nu \mu}=\frac{1}{4 \pi} \sum_{\mu=1}^{\mu=4} F_{\nu \kappa} F_{\kappa \mu}+\frac{1}{16 \pi} \delta_{\nu \mu} \sum_{\kappa=1}^{\kappa=4} \sum_{\lambda=1}^{\lambda=4}\left(F_{\kappa \lambda}\right)^{2}
$$

where $\delta_{\nu \mu}$ denotes the so-called Kronecker symbol, and where $F_{\nu \kappa}$ is the well known STR electromagnetic field tensor given in its covariant form with the Einstein-Lorentz metric convention by

$$
F_{\nu \kappa}=\left(\begin{array}{cccc}
0 & \frac{1}{c} E_{x} & \frac{1}{c} E_{y} & \frac{1}{c} E_{z} \\
-\frac{1}{c} E_{x} & 0 & -B_{z} & B_{y} \\
-\frac{1}{c} E_{y} & B_{z} & 0 & -B_{x} \\
-\frac{1}{c} E_{z} & -B_{y} & B_{x} & 0
\end{array}\right)
$$

The EMT, $T_{\nu \mu}$, for electromagnetic fields is then, according to the above requirement, shown to be given by (see [6], [4])

$$
T_{\nu \mu}=\left(\begin{array}{cccc}
u & \frac{S_{x}}{c} & \frac{S_{y}}{c} & \frac{S_{z}}{c} \\
\frac{S_{x}}{c} & \sigma_{x x} & \sigma_{x y} & \sigma_{x z} \\
\frac{S_{y}}{c} & \sigma_{y x} & \sigma_{y y} & \sigma_{y z} \\
\frac{S_{z}}{c} & \sigma_{z x} & \sigma_{z y} & \sigma_{z z}
\end{array}\right)
$$

where the Poynting flow $S$ is given by

$$
\boldsymbol{S}=\frac{c}{4 \pi}[\boldsymbol{E} \times \boldsymbol{B}]
$$

The energy flow $\boldsymbol{j}$ would then be given by

$$
\boldsymbol{j}=\frac{1}{4 \pi c}[\boldsymbol{E} \times \boldsymbol{B}]=\frac{1}{c^{2}} \boldsymbol{S}
$$

and the energy density $u$ by

$$
u=\frac{1}{8 \pi}\left\{E^{2}+B^{2}\right\}
$$

Thus furthermore the remaining tensor components $\sigma_{i k}$ of the above mentioned tensor $T_{\nu \mu}$ are given by

$$
\begin{aligned}
\sigma_{x x} & =\frac{1}{4 \pi}\left[E_{x}^{2}+B_{x}^{2}-\frac{1}{2}\left(E^{2}+B^{2}\right)\right] \\
\sigma_{y y} & =\frac{1}{4 \pi}\left[E_{y}^{2}+B_{y}^{2}-\frac{1}{2}\left(E^{2}+B^{2}\right)\right]
\end{aligned}
$$




$$
\begin{gathered}
\sigma_{z z}=\frac{1}{4 \pi}\left[E_{z}^{2}+B_{z}^{2}-\frac{1}{2}\left(E^{2}+B^{2}\right)\right] \\
\sigma_{x y}=\sigma_{y x}=\frac{1}{4 \pi}\left[E_{x} E_{y}+B_{x} B_{y}\right] \\
\sigma_{y z}=\sigma_{y z}=\frac{1}{4 \pi}\left[E_{y} E_{z}+B_{y} B_{z}\right] \\
\sigma_{z x}=\sigma_{x z}=\frac{1}{4 \pi}\left[E_{z} E_{x}+B_{z} B_{x}\right]
\end{gathered}
$$

In order to apply this EMT within the general frame of cosmology we need to pay attention to the general conditions under which the cosmological field equations generally are derived, namely conditions for a highly symmetrized universe which can be adequately described by the Robertson-Walker symmetry, i.e. by the so-called Robertson-Walker geometry where no direction and no 3D- space-point is preferred. This implies that mono-directional radiation fields cannot be a direct subject of these symmetrized cosmologies, and therefore when applying the above to cosmology we have to try to adapt the above derivations to the case of isotropic and homogeneous radiation fields.

\section{The Isotropic Cosmic Radiation Field}

We now assume that an isotropic electromagnetic, space-independent cosmic radiation field prevails at each place in the universe with the property

$$
\langle\boldsymbol{S}\rangle=\int^{2} \boldsymbol{S} d^{2} \Omega=\int^{2} \boldsymbol{S} \sin \vartheta d \vartheta d \varphi=c^{2} \int^{2} \boldsymbol{j} \sin \vartheta d \vartheta d \varphi=\mathbf{0},
$$

as e.g. given in the case of the cosmic microwave background radiation (CMB), and thus, for that isotropic radiation field, one can procede with the above derived tensor components by evaluating the space-angle-averaged tensor quantities $\left\langle T_{\nu \mu}\right\rangle$ and thus obtains the EMT in the following form:

$$
\left\langle T_{\nu \mu}\right\rangle=\left(\begin{array}{cccc}
u & 0 & 0 & 0 \\
0 & \left\langle\sigma_{x x}\right\rangle & \left\langle\sigma_{x y}\right\rangle & \left\langle\sigma_{x z}\right\rangle \\
0 & \left\langle\sigma_{y x}\right\rangle & \left\langle\sigma_{y y}\right\rangle & \left\langle\sigma_{y z}\right\rangle \\
0 & \left\langle\sigma_{z x}\right\rangle & \left\langle\sigma_{z y}\right\rangle & \left\langle\sigma_{z z}\right\rangle
\end{array}\right)
$$

Now furthermore one obtains for the components with $1 \geq \nu \geq 3 ; \mu \neq 4$ : the following expressions

$$
\begin{gathered}
\left\langle\sigma_{x x}\right\rangle=\frac{1}{4 \pi}\left\langle E_{x}^{2}+B_{x}^{2}-\frac{1}{2}\left(E_{x}^{2}+E_{y}^{2}+E_{z}^{2}+B_{x}^{2}+B_{y}^{2}+B_{z}^{2}\right)\right\rangle \\
\left\langle\sigma_{y y}\right\rangle=\frac{1}{4 \pi}\left\langle E_{y}^{2}+B_{y}^{2}-\frac{1}{2}\left(E_{x}^{2}+E_{y}^{2}+E_{z}^{2}+B_{x}^{2}+B_{y}^{2}+B_{z}^{2}\right)\right\rangle \\
\left\langle\sigma_{x x}\right\rangle=\frac{1}{4 \pi}\left\langle E_{z}^{2}+B_{z}^{2}-\frac{1}{2}\left(E_{x}^{2}+E_{y}^{2}+E_{z}^{2}+B_{x}^{2}+B_{y}^{2}+B_{z}^{2}\right)\right\rangle \\
\left\langle\sigma_{x y}\right\rangle=\left\langle\sigma_{y x}\right\rangle=\frac{1}{4 \pi}\left\langle E_{x} E_{y}+B_{x} B_{y}\right\rangle=0 \\
\left\langle\sigma_{y z}\right\rangle=\left\langle\sigma_{z y}\right\rangle=\frac{1}{4 \pi}\left\langle E_{y} E_{z}+B_{y} B_{z}\right\rangle=0 \\
\left\langle\sigma_{z x}\right\rangle=\left\langle\sigma_{x z}\right\rangle=\frac{1}{4 \pi}\left\langle E_{z} E_{x}+B_{z} B_{x}\right\rangle=0
\end{gathered}
$$

Furthermore when reminding that for an isotropic electromagnetic radiation field for time-averages over periods of $\tau=\lambda / c$ one finds 


$$
\left\langle\bar{E}_{i}\right\rangle=\left\langle\bar{E}_{j}\right\rangle=\left\langle\bar{B}_{i}\right\rangle=\left\langle\bar{B}_{j}\right\rangle
$$

(i.e. as much energy is in the magnetic and in the electric 3D-field modes!) and also considering that

$$
\begin{array}{r}
\left\langle E_{x}^{2}\right\rangle=\left\langle E_{y}^{2}\right\rangle=\left\langle E_{z}^{2}\right\rangle=\frac{1}{4 \pi} E^{2} \int_{0}^{2 \pi} \int_{0}^{\pi / 2} \cos ^{2} \vartheta \sin \vartheta d \vartheta d \varphi= \\
\frac{1}{2} E^{2} \int_{0}^{\pi / 2} \cos ^{2} \vartheta \sin \vartheta d \vartheta=-\frac{1}{2} E^{2} \int_{1}^{-1} \cos ^{2} \vartheta d \cos \vartheta= \\
\frac{1}{2} E^{2} \frac{1}{3} \int_{-1}^{+1} d \cos ^{3} \vartheta=\frac{1}{3} E^{2}
\end{array}
$$

will then lead us to the following results

$$
\begin{gathered}
\left.\left\langle\sigma_{x x}\right\rangle=\frac{E^{2}}{4 \pi}\left[\frac{2}{3}-1\right)\right]=-\frac{2}{3} \frac{E^{2}}{8 \pi}=-\frac{1}{3} u \\
\left\langle\sigma_{y y}\right\rangle=-\frac{1}{3} u \\
\left\langle\sigma_{z z}\right\rangle=-\frac{1}{3} u \\
\left\langle\bar{E}_{i}^{2}\right\rangle=\left\langle\bar{B}_{j}^{2}\right\rangle=(4 \pi / 3) u
\end{gathered}
$$

With these we are then led to the following cosmic EMT for $\tau$-averaged, isotropic monochromatic electromagnetic waves

$$
\left\langle T_{\nu \mu}\right\rangle=\left(\begin{array}{cccc}
u & 0 & 0 & 0 \\
0 & \sigma_{x x} & 0 & 0 \\
0 & 0 & \sigma_{y y} & 0 \\
0 & 0 & 0 & \sigma_{z z}
\end{array}\right)=\left(\begin{array}{cccc}
u & 0 & 0 & 0 \\
0 & -\frac{u}{3} & 0 & 0 \\
0 & 0 & -\frac{u}{3} & 0 \\
0 & 0 & 0 & -\frac{u}{3}
\end{array}\right)
$$

This is still not the final tensor because we must consider first the meaning and the cosmologically correct form of the diagonal elements of $T_{\nu \mu}$. While $u$ is simply the energy density of the EM field, the terms $-u / 3$ obviously reflect the wave pressure. To describe the action of the wave pressure we recall the cosmological principle. The required symmetry requires that for each mode of the existing EM-field a corresponding counter-mode should be existing whatever is its generator just to avoid any preferences. Therefore, simply as a request of the cosmological principle, one could then subdivide the universe into many sub-universes repeating each other in all dimensions. Hence considering the border of such a sub-universe as reflector of the considered wave modes, one would have a corresponding reflection of identical modes on both sides of this sub-universe. Under these conditions one can see that the action of the pressure of the reflected wave modes cancels and does not need to be taken into account. Thus, $T_{\nu \mu}$ for cosmic electromagnetically radiation fields can be written as:

$$
\left\langle T_{\nu \mu}\right\rangle=\left(\begin{array}{llll}
u & 0 & 0 & 0 \\
0 & 0 & 0 & 0 \\
0 & 0 & 0 & 0 \\
0 & 0 & 0 & 0
\end{array}\right)
$$




\section{Friedmann Equations for the EMT}

The above derived tensor $T_{\nu \mu}$ is - from a mathematical point of view - identical with the energy-momentum tensor of a pressure-less pure matter dominated universe (simply replace the quantity $u$ by $\rho c^{2}$, with $\rho$ the matter density and $c$ the velocity of light). The corresponding Friedmann equations hence are very simple and well-known:

$$
\frac{\dot{S}^{2}}{S^{2}}=\frac{8 \pi G}{3 c^{2}} u
$$

and

$$
\frac{\ddot{S}}{S}=-\frac{4 \pi G}{3 c^{2}} u
$$

With $S$ being the scale of the universe we now try to find a solution for $u=u(S)$. The first derivative of Eq.(30) is given by:

$$
\frac{2 \dot{S} \ddot{S} S^{2}-\dot{S}^{2} 2 S \dot{S}}{S^{4}}=2 \frac{\dot{S} \ddot{S}}{S^{2}}-2 \frac{\dot{S}^{3}}{S^{3}}=2 \frac{\dot{S}}{S}\left(\frac{\ddot{S}}{S}-\frac{\dot{S}^{2}}{S^{2}}\right)=\frac{8 \pi G}{3 c^{2}} \dot{u}
$$

which can be written as:

$$
\frac{\ddot{S}}{S}=\frac{4 \pi G}{3 c^{2}} \dot{u} \frac{S}{\dot{S}}+\frac{\dot{S}^{2}}{S^{2}}
$$

Now we replace the last term of Eq.(33) by Eq.(30) and get:

$$
\frac{\ddot{S}}{S}=\frac{4 \pi G}{3 c^{2}} \dot{u} \frac{S}{\dot{S}}+\frac{8 \pi G}{3 c^{2}} u
$$

This must be identical with Eq.(31):

$$
\frac{4 \pi G}{3 c^{2}} \dot{u} \frac{S}{\dot{S}}+\frac{8 \pi G}{3 c^{2}} u=-\frac{4 \pi G}{3 c^{2}} u
$$

Simplification results in:

$$
\dot{u} \frac{S}{\dot{S}}+3 u=0 \Longleftrightarrow \frac{d\left(u S^{3}\right)}{d t}=0
$$

Using

$$
\dot{u} \frac{S}{\dot{S}}=\frac{d u}{d t} \frac{S}{\frac{d S}{d t}}=\frac{d u}{d S} S
$$

finally leads to:

$$
\frac{d u}{d S} S+3 u=0 \Longleftrightarrow \frac{d\left(u S^{3}\right)}{d S}=0
$$

The one and only solution of Eq.(38) is:

$$
u=u_{0} \frac{S_{0}^{3}}{S^{3}}
$$

meaning that the energy density $u(S)$ of cosmic freely propagating photons scales with $1 / S^{3}$, in contrast to the conventionally assumed scaling with $1 / S^{4}$. 


\section{Conclusions}

We have studied in the foregoing sections of this paper the behaviour of universes with no other energy content but electromagnetic wave energy, i.e. no baryonic or dark matters are considered as cosmic energy carriers and no vacuum energy is considered here (i.e. $\Lambda=0$ !). Different from the usual thermodynamically guided considerations we, here in this paper, do not need to start from the assumption that a Planck spectrum prevails at the time of recombination of protons and electrons, which then afterwards is transported through cosmic time up to the present day epoch in an expanding universe. The latter assumption usually allows to describe the electromagnetic energy density in terms of Planck'ian relations between the photon energy density and the photon pressure. Together with the theory of cosmological redshifts of freely propagating photons (see e.g. [3]) this leads to the result that the energy density of the primordial cosmic background radiation preserving its Planckìn radiation character falls off with the inverse of the forth power of the cosmic scale $S$ (i. e. according to $1 / S^{4}$ ).

In this paper, however, we start our considerations - not presuming as usually done - that the electromagnetic cosmic photon spectrum has a Planckìn character allowing the application of well known thermodynamic relations, - but we allow for an arbitrary photon spectrum prevailing at some cosmic time $t=t_{0}$. To describe the cosmological behaviour of this photon spectrum we derive the EMT, $T_{\nu \mu}$, of this arbitrary radiation field starting from the electromagnetic field tensor $F_{\nu \mu}$ and find that the tensor elements of the tensor $T_{\nu \mu}$ are given by contributions from the Poynting flow $\boldsymbol{P}$, the energy flow $\boldsymbol{j}$, and the energy density $u$ of the electromagnetic field.

This tensor $T_{\nu \mu}$ we are then subjecting to the requirements of cosmic radiation fields which accordingly respect the needs of the cosmological principle (i.e. no space point and thus no space direction of wave propagation should be preferred for these radiation fields). Taking thus care of the isotropic character of the allowed radiation field leading to the fact that the space-angle integrated electromagnetic energy flows vanish everywhere, then causes a strong simplification of the radiation-induced EMT reducing the latter in a first step to the form of a main axis tensor with the spatial tensor components representing the negative radiation pressure. After some discussion on the meaning of radiation pressure of a homogeneous, isotropic cosmic radiation field we come to the conclusion that these pressure-induced terms have to vanish, and that one is finally left with an EMT in the form given in Equ. (29) which only contains as a non-vanishing term the time-like main axis component $T_{00}=u$.

With this form of the energy momentum tensor we enter into the Friedman cosmological field equations and interestingly enough can then show that the only remaining function $u=u(S)$ describing the energy density of the cosmic radiation field in a Robertson-Walker universe has to drop off with $u(s)=u_{0}\left(S / S_{0}\right)^{-3}$. This now confirms our earlier result ([1], [2]) derived with an independent photon-based concept. Hereby now we can confirm that both concepts, the photon concept and the wave concept both lead to the same result, namely that the energy density of the cosmic photon field drops off like the matter density also does, namely like $S^{-3}$.

\section{References}

1. H.J. Fahr and M. Heyl, "How are Cosmic Photons Redshifted?", AdAp, vol. 2, no. 1, pp. 1-7, 2017

2. H.J. Fahr and M. Heyl, "How are Cosmic Photons Redshifted?", AdAp, in press, 2018

3. H.F.M Goenner, Einführung in die Spezielle und Allgemeine Relativitätstheorie. Spektrum Akademischer Verlag, Heidelberg, 1996

4. R. Becker and F. Sauter, Theorie der Elektrizität, Band 1. Teubner Verlagsgesellschaft, Stuttgart, 1962

5. R.C. Tolman, Relativity, Thermodynamics and Cosmology. Dover Publications, New York, 1987

6. L.D. Landau and E.M. Lifschitz, Lehrbuch der Theoretischen Physik, Band 4. Akademie Verlag, Berlin, 1986 